\title{
An affective ephemeral social network for vehicular scenarios
}

\author{
Rasool Esmaeilyfard * and Faramarz Hendessi \\ Department of Electrical and Computer Engineering, Isfahan University of Technology, 8415683111, Isfahan, \\ Iran
}

\begin{abstract}
Spending time in the heavy traffic emerged in metropolises is an important part of people's daily routine. This situation could turn to an opportunity for a unique type of social communication among people. However, this social communication has its requirements and constraints. In this paper, the idea of an affective ephemeral social network for vehicular scenarios is introduced and the major components of the network architecture including affective individual profile, social matching and social group management are also described. Actually, the idea of matching user's mental model with its affective profile and matching these profiles with each other is accordingly one of the key points of this paper. In this regard, the privacy of the users is also considered and a prototype based on the affective cues has been developed for these concepts. Finally, a user study to investigate the subjective aspects and the user experience of the system is conducted.
\end{abstract}

Keywords: Ephemeral social networking, vehicular ad-hoc networks (VANET), interface design, affective profile, experimentation, human-computer interaction

\section{Introduction}

Social networks have provoked a real revolution in providing the basic human need to communicate and thus have been pointed out by many researchers as the key factor of a "new era of wonder for science". Moreover, after home and workplace, vehicles are the third place where a regular citizen spends his time [1]; Owing to this significant amount of time, cars have become an integral part of everyday life. On the other hand, the heavy traffic in the metropolises provides suitable condition for the vehicles to be with each other for a while. As the electronic devices are widely and extensively used in vehicles, a new generation of users is looking for more effective use of them and interact with the environment. In this situation, different people with different tastes and social conditions are gathered together in the same location. The absence of users' prior knowledge about each other and also the lack of face-to-face interaction among a large number of users provide an appropriate basis for the appear-

*Corresponding author. E-mail: resmaeily@gmail.com. ance of people's favorite character. In fact, each person can express his viewpoints without the fear of his privacy and social limitations. Moreover, there is a lot of wasted time in the traffic that can be used for this purpose. Lastly, the environment itself and the applied stress can make different moods to be appear in the users. To the best of our knowledge, these conditions are unique to the urban traffic and the proposed social network is perfectly matched to the vehicular scenarios.

Additionally, the sense of being together and the opportunity for communication among users are limited to the time the vehicles commute with each other in the traffic. The temporariness of this communication network brings about a great deal of limits and restrictions for developing social ties which causes initiating a communication process would become quite difficult to actualize [2]. However, by changing the circumstances and expanding the viewpoints, these limitations will be reduced; potential benefits for communication between vehicles will be realized and a sense of "presence" (being there) and "co-presence" (being there along with others) will be appreciated. 
However, little information exists concerning a social vehicular network from the human-computer interaction (HCI) perspective. In this paper, an identification mechanism for this network is introduced concerning this issues. This identity should consider privacy and rapid identification. In this way, the social network service architecture is introduced from the identity selection and social matching mechanism to social group management in vehicular scenarios. The paper shows how the affective cues are efficiently used to improve human-computer interaction in these scenarios and facilitate the communication process.

The subsequent parts of this paper have been organized as follows. In Section 2, some related works is outlined. Section 3 presents the motivation for an ephemeral social vehicular network. Next, the proposed approach is reviewed in Section 4 and an introduction to the network architecture is presented in Section 5. Section 6 continues with describing the prototype. Section 7 presents the user study and its results. Finally the discussion and conclusion will appear in Sections 8 and 9 respectively.

\section{Related work}

\subsection{Socializing car}

Social networking is considered as a remarkably fascinating phenomenon to many scholars. However, in spite of a great deal of research on two-way interactions or sharing contents of interest between vehicles, little is known about opportunities that a social vehicular network could bring for individuals. Over the past few years, in-vehicle information systems have not undergone a significant change. The most important of these systems was based on an exchange of textual information [3,4], making voice and video calls [5], creating an entertaining environment inside the cars [6] as well as navigating and commanding vehicle system [7]. The limitations imposed on this type of communication will cripple the joy in vehicle environment or participation in collective works and decision-making. Schroeter, et al. [8] mentioned an innovation hotspot for new social applications and services to interact with the others surrounding the car. Moreover, Alt, et al. [9] studied how a driver uses the opportunity for waiting times in front of the traffic light to start interaction. The interaction between vehicles has also been investigated by Lee, et al. [10]. They implemented a virtual "flea market" called FleaNet in order to find a customer/vendor with matching needs/resources. Lequerica, et al. [1] considered the technical challenges in designing the vehicular social networks but they did not take the social network users and their relations into account. However, this work not only form an ephemeral social vehicular network, but also considers social communication barriers and infuses a sense of familiarity with respect to privacy.

The potential of social networks in the vehicle has influenced some companies to add basic social features to navigation applications and services based on the internet. For example, Aha (http://www.aharadio.com) is an application that adjusts web content, messages and traffic reports to the vehicle environment. It is basically a personalized radio with interactive features that turns the web contents and messages from existing social networks into audio streaming. Waze (http://www.waze.com) as a navigation and GPS system enables the drivers to exchange the traffic information and report traffic incidents. The information posted is only valid for a period of time and geographically referenced. Most of the other existing applications like Navigon, ALK Technologies, Telmap \& GyPSii primarily allow their users to post location and route information to their contacts in existing social networks (mainly Facebook and Twitter). The affective requirements and social barriers of the vehicular social networks based on the VANET are considered in this study.

\subsection{Mobile social networks}

To the best of our knowledge, no specific architecture has been proposed for social vehicular networks and the current ones mainly focus on the mobile social networks. The most comprehensive ones are as follows: The SAMOA [11] enables the mobile users to create roaming social networks by detecting the neighbors with desired interests. Although SAMOA provides an open framework, the inference method is not consistent with the vehicles' high mobility. MobiSoc [12] collects the people/place profiles and location metadata in a centralized server. Several specific modules discover and represent the relationships between persons and places. MobiClique [13] provides an specific ad-hoc network middleware through communications with neighbors using opportunistic communication. Moreover, the profiles' initial data is synchronized with online social networks like Facebook. Yarta middleware [14] is a user-centric mobile social network middleware that attempts to introduce Mobile 
Social Ecosystem as a set of interactions between users and devices in an specific physical location through introducing independent entities. Considering the features of these middleware, a middleware for vehicular scenarios is proposed in this paper.

\subsection{Avatars}

Affective identity can be looked in different ways. One part of the proposed solution is using avatars, as will be discussed in detail in Section 5.1. Many researchers have investigated avatars and their roles, positions and effects in different virtual environments. For example, Wallace and Maryott [15] studied the role of avatars in the virtual world, their contribution in increasing a sense of presence and participation and also reducing the negative effects of such a presence. The possibility of interaction and non-verbal expression in networked environments from various technical aspects was evaluated by Hamilton [16]. Sung, et al. [17] and Messinger, et al. [18] assessed the personality differences between the actual self and the avatar self and the correlation between these two. Prince [19] analyzed the avatar identity and its impact on understanding and discovering new ways of positioning identity projection. Chen, et al. [20] examined the role of abstraction in the presence awareness and achieving the desired goals. Additionally, Bente, et al. [21] addressed the role of visual and non-verbal behavior in interpersonal trust and communication quality. These studies was taken in mind and present an opportunity to produce and deliver affective cues of the users to facilitate social communications.

\subsection{Affective profile}

Affective computing researchers have been making a lot of effort to model human psychological aspects since the 70s. Some of their achievements to implement what they believe to be lifelike agents are [2225]. Their idea is to model lifelike believable characters with personalities, goals and human-like emotions while people have psychologically answered to interactive computers as if they were humans. This issue directly lead to consistency, coherence, and predictability in computer emotional reactions and responses [23]. In this way, many researchers have worked on the problem of how to express the relationship between psychological aspects. For example, [24] described a scheme to represent psychological aspects to be used in the design of socially intelligent artificial agents. This scheme is composed of a taxonomy of affection, mood, emotion, and personality. Affective profile is elicited with the help of the taxonomy presented in [24].

But in order to elicit the affective information quickly, different approaches are presented in the literature so far. For instance, Gosling [26] presented an extremely brief personality inventory named TIPI test. TIPI (Ten-Item Personality Inventory) is composed of 10 items based on the Big Five factors. Moreover, according to Russell $[27,28]$ emotion could be extracted from a valence (i.e. pleasure, positive versus negative affect), arousal (low versus high level of activation), and dominance space (the degree of control over the emotion) [29]. On this basis, Broekens and Brinkman [30] tried to obtain the user emotion in different aspect with an AffectButton. Their work shows that eliciting these information in a summarized and visual form is feasible. Considering these studies, the proposed affective profile is formed visually and briefly in this paper.

\subsection{Communication infrastructure}

Regarding VANET communication, various methods and protocols have been proposed for group formation and data exchange [31,32]. Most of these methods are based on techniques, such as Centralized [33], Group/Cluster based [34], and Hierarchical [35]. Furthermore, many of the dissemination protocols for VANETs are based on the following techniques: flooding, broadcasting, neighbor knowledge based exchange, and cluster based approach [36]. Recently, new methods is also provided to access various resources during mobility such as Vehicular Mobile Cloud [37,38]. Fan [39] considered a simple scheme to form clusters and proposed an efficient broadcast method. This approach is used as the base algorithm and social-head election procedure is added to meet the solution needs.

\section{Motivation}

This ephemeral social vehicular network has the potentials to host many applications. The following examples clarify this issue. The users could form a team to play in groups, transfer their feelings to the users and prevent aggressive, selfish and anti-social driving behavior while enjoying the other users' sympathy and support [8]. Moreover, collective decision making process shall be improved among the users using the social information of the others. In fact, the users could 
reach to the same conclusion easier with the users that have similar feelings and attitudes or have sympathy with them [40]. For example, the users could make collective decisions to mark the vehicles with high risk behavior. They can also assign rewards for friendly driving vehicles which allow for an overtake or offer parking space. Social and emotional information may also have other positive impacts. For example, users based on the current surrounding situation could be encouraged to share information with others or use their mutual service like shared music or a $3 \mathrm{G}$ line to establish an internet connection [41].

These are just some of the examples to explain the benefits of this ephemeral social network and ephemeral social knowledge. For instance, in designing various algorithms, this method could be used to provide the incentive required to perform different tasks [42] or reduce the false positive error rates in intrusion detection systems [43] etc.

\section{Approach overview}

The users' communication in this social network is limited to the time that the vehicles are stopped or moving with the low speed over common paths like a highway. Thus, simplifying the interaction with this social networks from the initial configuration, registration, tune and usage has a significant impact on its effectiveness. Besides, the lack of familiarity of users with each other causes difficulty in initiating a communication process [2]. Applying an appropriate schema to facilitate communication and reduce social differences is essential.

In this regard, a user in this network chooses an ephemeral identity and presents its desired personality and characteristics through it. This identity is based on a fitting avatar and extending its affective cues like personality traits, emotions and preferences. Special attention is paid to the selection process and the relevancy of the information to reduce the profile selection time. The approach in the next section is considered on two phases of affective individual profile and affective social matching.

Considering the affective cues of the profiles, in this study, it is focused on how the constructive nature of human identity and preferences can be supported in an interface. In this regard, various issues charge the application designers with new challenges such as design for mobility, design for a wide audience with various levels of adequacy in the use of new technologies or the experience of using the similar applications [44].

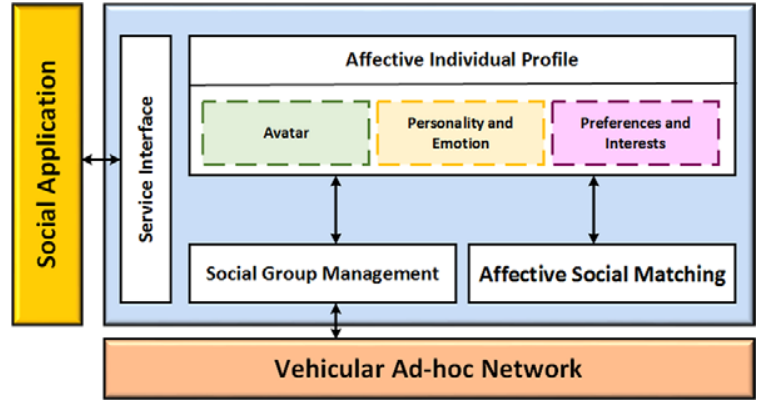

Fig. 1. Architecture.

In other words, the design of an elegant and pleasing interface is important, however, the success of the system is based on perceiving information in an intuitive and easy way [45].

After presenting the context of this social network, it is needed to measure users' experience with this system. Specifically, the quality of the affective profiles or affective social matching is not the only interesting factor. The users' satisfaction with the system as a whole is also important. In this study, it is hypothesized that the proposed mechanism could be a good tool for introduction and social matching. In this way, this method is evaluated.

The lack of fixed infrastructure between vehicles requires a suitable method for transferring information between them. In this paper, an appropriate method is presented to form the social groups and prepare the ground to apply the network policies.

\section{The architecture}

The described ephemeral social vehicular network is provided by a service on top of the VANET platform. The architecture of this network is consist of five components over the VANET - Affective Individual Profile, Affective Social Matching, Social Group Management and Service Interfaces.

This architecture (Fig. 1) is a generalized version of that of middleware, proposed by Bellavista, et al. [46] to consider the ephemeral social network requirements for vehicular scenarios. A full description of the service is presented below.

In order to establish the user identity, each user presents himself by the Affective Individual Profile. This component helps the users to present an initial introduction through affective cues such as emotions, personalities and preferences. The key objectives followed by the creation of an affective profile have been 
clearly predicted in this component, which are simple introduction and tuning, abstraction, anonymity, adaptability to expected identity, daily emotions, preferences, conditions and environment.

The exchange of information in the social network is processed through formation of a group and registration in the network. In order to achieve optimal management of these groups, the Social Group Management component develops mechanisms for registration, group formation process and data exchange protocols over the VANET.

In addition to establishing a profile for the user, matching the users to each other and providing proper recommendation in this regard are essential for the effective use of the network. The Affective Social Matching component provides this ability for the user to match themselves with their goals and backgrounds. This component plays a significant role in creating an adaptable and personalized system.

A social application can access the social network and profiles of users through an interface to gain the acquired knowledge at their disposal.

\subsection{Affective individual profile}

As noted, creating a virtual world in the traffic space gives the people the opportunity to create their own identity and introduce it to the others. However, this identity should be presented in a way that provides the possibility of understanding, evaluating and acting towards any person [47]. In todays disembodied world of the virtual world, many attributes required in connection with the person's personality and psychological aspects do not exist [48].

The intended affective profile should assist users to achieve their goals quickly, communicate and highlight their aims and preferences enjoying the transition of their abstract views. This will allow them adjust to a new identity and feel better with the anonymity that can be found in place, somehow difficult in loyal identification. The anonymity is the most essential factor for this issue. This ephemeral social network can easily benefit from this factor due to using computermediated communication. Actually, an affective individual profile could prepare the prerequisites to overcome the psychological and social hurdles of the realworld and prepare the circumstances for the adjustment of the personalities and preferences to establish an initial relationship with the others.

Smart systems need accurate information models to be efficient. An affective characteristic and preference elicitation interface needs to extract information from the user's mental representation of that information and translate it into a representation that the system can reason with. Thus, affective individual profile modeling entails three components: mental representation, elicitation and the system's profile representation.

In this system, the users choose a role or character by choosing an abstract avatar and complete its affective characteristics.

In the following sections, different parts of an affective individual profile is discussed and modeled. Additionally, a method is presented to elicit these information.

\subsubsection{Avatar}

In order to simplify the process of identifying and communicating with other users and provide cues about their own aspects of personality, each profile is initiated around the context of an avatar. This avatar does not only substantiate the user's identity in the network but it can also facilitate the statement of feelings and emotions through non-verbal expressions. It accordingly aids to build social interactions. This type of characterization admits users to be independent from any physical and social situations (e.g. age, gender, social status) and to select one or more different identities in this new world.

Although avatar characterization may look simple, recent studies in the field of avatars reveal that this could provide an opportunity to produce and deliver a demonstration of our real self [49]. As stated by Turkle [49], "people are able to build a self by cycling through many selves". In fact, Turkle argues that avatars put forward an opportunity to form our identity. In this case, even when a vehicle is acting a part, it is not separated from self, but belongs to a developed and expanded variety of self [16]. Thus, if we look at them through this perspective instead of through a mask that conceals our identity, we find them as an opportunity to probe some aspects of our self, i.e. risk-taking, curiosity etc. which might be unfeasible to do in our everyday lives.

With the widespread development of everyday means of communication such as ubiquitous computing and computer-mediated communication, the role of avatars has been considerably extended. At the same time, some analysts have asserted as no clear-cut difference between a physical and virtual presence can be clearly determined. Actually, as the social interaction between our real self and our avatars is going on, insistence on their separation would be replaced on a 
dominant correlation between them [17]. Therefore, as we role-play in the part of an avatar, like a character in a computer game; this relationship could be evolved in these contexts, through our conducts and it could progressively close our real self and our avatar together.

More importantly, there is a variety of self-projection that a user is disposed to present in different social situations. As we might change our looks with clothes, shoes etc. - depending on who we are communicating with, the activity we are handling, the context we are associating with and the aspects of identity we would like to focus on - we might lean towards employing different avatars or various of them to create a sort of visual communication, to affect our audiences and also to influence the purpose of interactions. Meanwhile, just in a few moments, we might introduce ourselves quite differently. In other words, an identity formation is a process influenced by responding to altering relationships and the changing world around us. Consequently, presenting an identity as well as identification with an avatar, should be inspected in the frame of dependency on the context, social continuity and flexibility [19].

A user might be presented by an avatar with varying degrees of similarity, produced by different means such as abstract shapes, objects or portraits, creatures which their realization could be symbolic, descriptive, arbitrary, or even conceptual. Currently, avatars are predominantly abstracted. Because, abstraction lead to develop an effective communication; a great deal of research in psychology support the concept that abstraction increases popularity, social presence and copresence instead of reducing them [20]. In fact, people have a tendency to communicate better with those who seem similar to themselves and this could result in a sense of familiarity. When an avatar is generalized, a sense of similarity instead of difference is spread [21]. In other words, too many details do not necessarily contribute to a more accurate identification and may reverse the effects. In addition, some social scientists have confirmed that even special characteristics of avatars demonstrate special characteristics of owners. For instance, in [18], it has validated that people with more attractive avatars than their real selves are more confidence in virtual worlds than in the real environments.

\subsubsection{Personality, emotions and preferences}

Following the former descriptions, an affective individual profile could provide reasonable ideas to the others with personality information and conveying emotions and preferences. In terms of psychological aspects, personalities (long term features), emotions (short life-time features), socio-cultural aspects, emotional intelligence and soft skills may be considered. The specific and instant requirements like preferences and competencies could be added to this list too. There is an agreement in the literature that the emotions and preferences of people are not always stable and consistent. In reality, the people construct them when necessary $[29,50,51]$. There are numerous views on this issue.

However, an easy, ready-to-implement method for designing interfaces for this purpose has not been presented yet although there have been few attempts in this regard like [30,52]. In this study, it is intended to elicit this kind of information visually and quickly to simplify the profile selection. Thus, in the next section, it is shown that a compact elicitation of this information is enough for social matching due to the prominence effect. This information will be provided to the others as a criteria to compare the characteristics and also transfer their feeling as mentioned in the Section 3.

\subsection{Affective social matching}

Users in this social network are social creatures. They look for other contacts in this ephemeral social network for a multitude of purposes as mentioned in Section 3. Therefore, finding appropriate persons according to the user's goals plays a major role in network effectiveness. Diversity of the people in this network could turn this requirement to a challenging task. Additionally, due to the limited time of communication between users in this ephemeral social network, an efficient recommendation and matching could lead to rapid communication between users. Therefore, considering these issues mechanisms should be designed to bring people together, shorten the process of social interaction and encourage the users to participate.

However, the objectives of social matching systems are incompatible with traditional recommender systems in two fundamental ways. They can propose particular opportunities to collaborate and bring the people together who don't already know each other but have shared interests [53]. Actually, the social matching systems try to comfort decision-making by identifying and proposing specific people according to the user's wishes and introducing them to ease interaction.

While computational systems are good at gathering and aggregating data, humans are highly capable of in- 
terpreting and integrating information representations and determining appropriate actions [54]. The psychological effects $[55,56]$ play a major role in achieving the goals of the users, e.g., loss aversion (the tendency of people to prefer avoiding losses to acquiring gains) or anchoring effects (relying too heavily on one piece of information).

Thus, the primary evaluation metric for a social matching system is whether the users realize their objectives or not in this system [57]. Some aims such as finding someone to answer a particular question are more information-oriented. Some aims like finding someone to play a particular game consider socializing, recreation or leisure. Hence, the goals of the user should be elicited and made explicit; consequently, the systems should act and be evaluated considering these goals. Fischer, et al. [55] and Sedikides, et al. [58] focused on the prominence effect and its correlation with the goals of the decision task. According to this effect, people lean towards a choice that is superior only on the most important attribute (the most prominent). Their results in three studies showed that the prominent attribute when the goal was selection between choices will be more heavily weighted than when the goal was to achieve at a matching value.

On this basis, the matching method is based on the prominent attribute and evaluated it later through the user study. In doing so, the users could choose an option from each category and put them in three different levels of ordering. In addition, a selected option in each category may assigned with an intensity. For example, if the selection is relevant to emotion, different levels of valence, arousal and dominance are assigned to the selection. The system matches the users based on the lexicographic ordering regarding these levels and the intensity.

\subsection{Social group management}

In the absence of a fixed network infrastructure for vehicles, establishing social groups and administering the social network rules are difficult. Thus, protocols to manage the exchange of information between vehicles and establish such an infrastructure seem to be vital and essential.

In order to create a communication infrastructure for this ephemeral social network, a convoy of vehicles or vehicles that commute with each other initiate a social group. In addition, these vehicles should be able to communicate with each other in one hop or multi hop transmission to be within one social group. Sev-

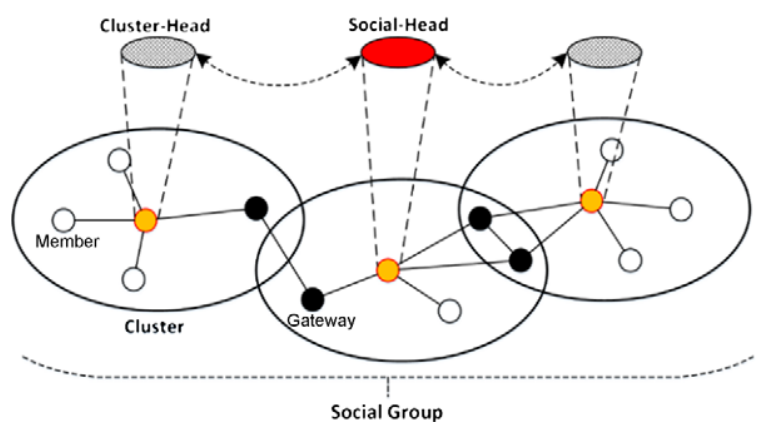

Fig. 2. Social group formation.

eral adjacent vehicles that can communicate with each other in one hop transmission form a cluster; clusters join each other to form a social group.

In order to validate data, apply the social network rules and disseminate the data through the chain of connected vehicles, a clustering method is needed to choose one of the vehicles as the social-head. Until now, a large number of studies have focused on the optimal methods for clustering nodes in VANETs [59-62]. However, an approach is needed to choose one of the vehicles as the social-head, collect all the data and broadcast them through the chain of connected vehicles. According to this requirement, a clustering method has been investigated recently by Fan $[39,63]$.

This method gives us the ability to choose the cluster-heads for smaller clusters so that it is possible create a hierarchical structure and choose one of the heads with suitable properties as the socialhead (Fig. 2). Basically, this method partition the network into a group of clusters. In each cluster, a single cluster-head manages all the other members in the cluster. Moreover, the gateways to the other clusters are formed by the non-cluster-head nodes with at least one neighbor belonging to other clusters. Therefore, as Fig. 2 illustrates, the social-head, cluster-head and gateways form the backbone of the network and provide an infrastructure to broadcast the messages through the social group as described by Fan [39].

This clustering architecture limits the communication relay points for each node to a small subset of the total network so that, each cluster head aggregates the local member topology and acts as a relay point for communication between its members and the members of other clusters.

At the beginning of this clustering algorithm, each node announces itself as a cluster-head by putting its own address and id in the beacon to be broadcasted. 
This id is a random value initially assigned to each node. This will enable its neighbors to have a full knowledge of its current neighbors and make decision whether to change its current cluster status or not. The status changes could be bi-directional either from the member node to the cluster-head or from the clusterhead to the member node.

Four principle factors contribute to change the status of a node including moving direction, leadership duration, projected distance variation and vehicle id. A node looks for a cluster-head from its "candidate pool" formed by all its neighboring cluster-head nodes with the same moving direction and a lower id. This node gives up its original status if it finds such cluster-head nodes existing around. Moreover, the pivotal points in choosing the best in the pool are the leadership duration, i.e. the period that a node has been a leader since the last role change and projected distance variation of all neighboring nodes over time which estimates a degree of its local topology changes. This implies that the longer the duration, the more stable the node will be. Therefore, the following rules could express the cluster-head election mechanism:

1) Initially, opt for the node with the longest leadership duration.

2) If all are the same, choose the node with the lowest projected distance variation of all neighboring nodes over time [63].

3) If all are the same, the one with the lowest id (LID) [64] will be selected as the cluster-head.

However, a social-head is necessary to be elected in the protocol. Similarly, each cluster-head and socialhead (if exists) prepares a packet including its vehicle id, leadership duration, volume of stored active data and its role; then the cluster-head disseminates it in regular intervals to the reachable clusters. Therefore, cluster-heads have a full knowledge of the cluster-heads of the social group; a "candidate pool" is formed, as well. If there is already one social-head in the pool, it will be elected as social-head; otherwise the social-head is elected in the pool once again using three factors of the volume of stored data, the leadership duration and lowest id because stability is pivotal in election. When a cluster-head chooses a social-head, the social-head id is broadcasted to their members and they must save the social-head id.

Furthermore, merging social groups could also cause the existence of two or more social-heads in the network. For example, a group of vehicles stops at a red light and a group behind them may move faster and join them. In this case, just one of the social-heads should remain and the other one will give up its role and send all of its data (which was obtained from acting in this role) to the other social-head. Since a major issue in social-head election could be the volume of the data stored in the social-head, in case a social-head observes another social-head in its pool, it should compare the three factors of stored data volume, longest leadership duration and lowest id and decides to give up or stay.

\section{Registration and exchange protocol}

Following social group formation, each vehicle must coordinate its social information with the socialhead. The architecture allows to define and apply rules and policies in the social-head. Hence, the users should register in the network. This will not only make the social network information accessible to the users with their credentials already assessed and registered in the network, but also permits the rules for registration and access levels to be designated as well as special usage policies to be applied.

Correspondingly after social group formation and social-head selection, a vehicle is supposed to send its affective individual profile to the social-head so as to join the social network. The social-head receives it and sends it to the other social group members. The social-head also sends all its maintained affective individual profile to this vehicle as soon as it joins the network.

However, in this social network vehicles could easily change their profiles and tune it in accord with the conditions. Additionally, the vehicles may depart the group. As a consequence, an expiration time is defined for each profile when the profile is invalid afterwards. Thus, each vehicle resends its profile to the social-head after a time $\mathrm{t}$.

\section{Assumptions}

Considering this social network requirements, it is assumed that the vehicles are connected to each other wirelessly using a low-power IEEE 802.11p interface, such as DSRC [65]. Each of the vehicles is also equipped with a low-power Wi-Fi access-point and bridge the user's data to the vehicular network and vice versa. Using the Wi-Fi access point in vehicles regarding its wide support in portable devices could enable the usage of the network for the passengers. Thus, the drivers and passengers could use a wide variety of devices like smartphones, tablets, personal navigator devices (PND), embedded on board units (OBU) or laptops to connect to this social network. Additionally, 

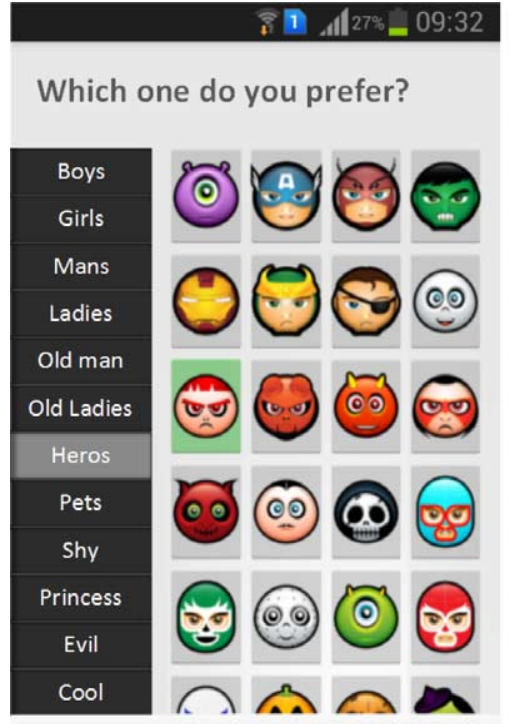

Interests Join the others!

a) Avatar Selection

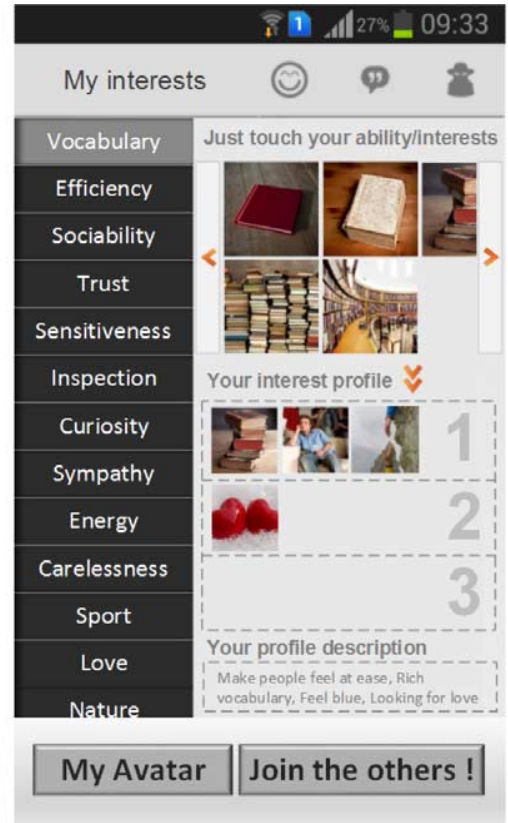

b) Preference selection

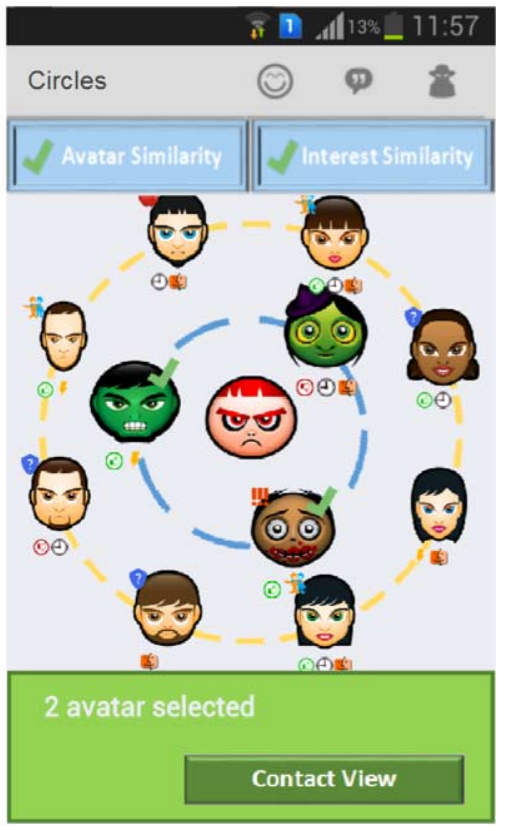

c) Circle view

Fig. 3. Prototype.

this access-point operates in low-power mode to prevent interference with other devices.

\section{Prototype}

Up to now, the ephemeral social network architecture is introduced. The issues related to the affective identity selection and social matching such as avatar, visual personality and preference selection have been discussed. In this section, the developed prototype to elicit and represent the affective profile is presented. According to the guidelines provided in [53,66-68], the user was involved in the design and it was implemented based on the user's feedback in several creative sessions. The detail of these session is not presented here to avoid verbosity.

The prototype was designed and built for touch phones running Android OS. This prototype allows the user to adopt an affective profile. Afterwards, the received profiles that matched with the owner profile are recommended. Purposefully, the major focus was on the identity selection and recommendation process. Due to lack of enough simultaneous participants and the absence of a vehicular network, the prototype was filled with preselected profiles as it will be discussed in the user study. All of the user interaction is logged in this system to be evaluated later.

\subsection{Identity selection}

The first point of user interaction with the system is selecting his affective profile to register in the network (Fig. 3a). The avatar is the first identifying information received from the user. Avatars are consists of $2 \mathrm{D}$ icons that a personality, mood or feeling is perceived from them and are classified consequently. By selecting an avatar the user is able to connect to the network. It has been shown in the previous section that an avatar could provide the minimum information necessary to establish the connection. In the following, the user could select its personality traits, emotions and preferences in various categories. These categories are focused on the standard personality traits like Big Five factors and the constructive nature of human preferences. As it is shown in Fig. 3b, these categories are rather unstructured and do not have a specific start and do not necessarily linked to each other. The user could touch an image from each category and add it to its sensationboard. He can do this step by step by selecting each category. Each sensationboard includes images that show a specific profile at a glance. Similar work has also been used in advertising to transfer an emotion. The user could also drag his interests between different levels of interest.

This information establish a profile for the user to register in the social network. This profile could pre- 
serve the privacy and provide the user's goals to the others with an appropriate abstraction. The visual elements help to do this simply and quickly; of course, this does not cover all types of possibly affective information. However, it was regarded as a suitable starting point for investigating the use of affective information in introduction and matching because it involves psychological and individual dimensions of profile that are important in this ephemeral social network.

\subsection{Circle view}

Once registered on the network, a view of the users around is displayed (Fig. 3c). In this prototype, due to lack of enough simultaneous participants in the test stage, this view is filled with pre-selected profiles to give a real imagination to the users. Each profile is presented using its avatar. These avatars are shown on circles around the user`s avatar. They may surrounded with icons highlighting the most degree of match or the first priorities. The user could select just the avatar similarity, interest similarity or both as the social matching mechanism. The avatar similarity mechanism matches the profiles based on the personality traits and the emotions assigned to each avatar. The interest similarity matches the profiles based on the traits and preferences selected considering the level of interest and intensity. If both of these factors considered and applied as the ranking method, the profiles that better match with the user profile are displayed in closer circle. Since the initial choices may not be enough or options do not match well, the users are free to tune it and reorder the choices. After the profile tuning, the profile resend to the network and the circles update respectively. During the evaluation, the satisfaction for this view and profile tuning is investigated.

This view also provides the possibility of initiating an interaction with the users. By touching each of the avatars, the avatar gets selected and the interaction could be started.

\subsection{Contact view}

In this view, a full view of the user profile and the ability to communicate with a user or users on the network are provided. Currently, some of the sample applications have predicted. But this application could be easily extended as it mentioned in the architecture. In the present, the user could share his playing music with the selected users, send text messages or files and invite them to the games. The history of interactions is also displayed in this view.

\section{Study}

In order to evaluate the proposed ideas and the considered hypotheses, the identity selection and the social matching procedure are investigated. It is inspected weather the proposed interface could give the users a satisfactory experience for introduction and recommend the suitable contact. The former statements including the ability of the profile to transfer the affective cues, the potential of the social matching method in achieving the users' goals and finally encouraging the user to participation are also explored. The participants in the experiment were allowed to interact with the designed prototype.

\subsection{Setup}

The participation takes place in two stages. First, some of the participants were invited to select their identities through the prototype (Section 7.2). Afterward, these identities used as input to the next stage which the participants were asked to select an identity and work with the system as a whole.

In order to inspect the hypotheses effects, a textual version of the prototype is prepared. In this prototype all of the characteristics are specified through textual selection in the same style as the original prototype and the users around are displayed in a list view. The first used prototype is also randomly assign to the participants and they are asked to use that to prevent ordering effect. However, the procedure in both systems are similar.

The user perceptions were incorporated to expand the explanatory power and robustness of the evaluations. In this investigation several objects of user experience were treated parallel to acquire more insight in the workings of user experience. Additionally, structural equation modeling (SEM) was used to conduct a mediation analysis on desired effects. SEM concurrently analyzes the robustness of the measured constructs and the relationships between them statistically. A detail review of this model is presented by Knijnenburg, et al. [69].

\subsection{Participants and procedure}

Participants were recruited from various segments of society in a city with heavy traffic including drivers, staffs, students as well as regular citizens. Invitation was only dedicated to those people who had experience with the touch phone to do a meaningful experi- 
ment. The test was taken in the vehicles including cars and buses in the traffic. The first stage was performed on 134 participants (76 male and 58 female) to create a profile using both prototypes. They asked to choose an identity and introduce their personality and preferences. The profiles were used in the next stage as builtin profiles. In the second stage, the participants were asked to choose an identity like the former stage. They also asked to consider the system recommendations, check the eligible profiles and rate them if they found it helpful. Hundred and Thirty four participants, $61 \mathrm{fe}-$ male and 73 male consented to participate in this stage.

Before starting this stage, these participants were asked to answer 11 questions about their daily conditions and personal habits (usage of the vehicles, familiarity with social networks and trust tendency). Questions were measured with a five point Likert scale or an unrestricted free-report response. They subsequently asked to start working with the first selected prototype. The participants had to enter to the first prototype, select an identity, check the recommendations and rate the desired profile according to the level of interest. They could optionally change their identity or tune it as desired. Next, the participant switched to the other prototype and did the former steps again. In addition, they could switch between prototypes alternatively and compare them. Ultimately, they were asked to fill a questionnaire with 27 questions.

The mean age of participants was 24.11 years old in the range of $18-31$ ( $\mathrm{SD}=4.12)$. Respondents were asked to specify the vehicles they used: $94.3 \%$ stated cars, $62.3 \%$ stated buses, $4.1 \%$ stated motorcycles, $3.9 \%$ stated bicycles and $0.02 \%$ stated trucks. In terms of daily usage of vehicles, the participants' responses ranged from 20 minute to 5 hours with an average of 1 hour and 12 minutes ( $\mathrm{SD}=32$ minute). Respondents were also asked the percentage of their time in traffic; $74.6 \%$ of respondents stated to spend at least 30 minutes in traffic with $51.7 \%$ reporting to spend more than 1 hour in traffic.

Nine participants who completed less than 25 percent of the questionnaire items were excluded; the rest of the participants answered the questions almost completely. The answers to 38 questions applied to a confirmatory factor analysis. In this analysis categorical indicators and a weighted least squares estimator are used and the following 8 factors are estimated:

Understanding the concept and functionality was measured using 3 items $(\alpha=0.91)$ rated on a Likert scale from 1 to 5 , with poles of strongly disagree to strongly agree. An instance item is
"The explained social network is easy to understand".

Reducing privacy concern in the network was measured using 4 items $(\alpha=0.83)$ rated on Likert scale. An instance item is "I am worried about abusing my personal information".

Efficiency of encouraging users to participate was measured using 4 items $(\alpha=0.79)$ rated on a Likert scale. An instance item is "Using this social network is interesting and exciting".

Perception of the social matching quality was measured using 6 items $(\alpha=0.81)$ rated on a Likert scale. An instance item is "The recommended items on the circles are worth to take a look at".

Making sense of presence and co-presence was measured using 6 items $(\alpha=0.72)$ rated on a Likert scale. An instance item is "I sense my feelings have an impact on the other users".

Realization of affective cues was measured using 5 items $(\alpha=0.89)$ rated on Likert scale. An instance item is "I feel impressed by checking the avatars and profiles".

Familiarity with social networks was measured using 3 items $(\alpha=0.74)$ rated on Likert scale. An instance item is "I access social network sites regularly".

Trust propensity was measured using 3 items ( $\alpha=$ $0.76)$ rated on Likert scale. An instance item is "People generally have the sense of sympathy and support and help the others".

The high cross-loading and/or high residual correlations and low communality of 6 items in questionnaires led to exclusion of these items. In addition, 3 items on the usage of the vehicles failed to converge to a stable factor. The values of average variance and Cronbach's $\alpha$ of the remaining factors were high and indicated discriminant validity.

\subsection{Results}

The 8 factors obtained along the logged behaviors of the users applied to a structural equation modeling which simultaneously qualify the structural relation between the factors and other variables. The model has a good fit, with a non-significant $\chi^{2}(17)=21.03$, $\mathrm{p}=.23, \mathrm{CFI}=.99$, RMSEA between 0 and $.033(90 \%$ $\mathrm{CI}=[.00$ to .074$]), \mathrm{SRMR}=.035$. The effects found with this model is displayed in Fig. 4 and factors are scaled to have an SD of 1.

The results show that identity selection and social matching both have independent positive effect on the 


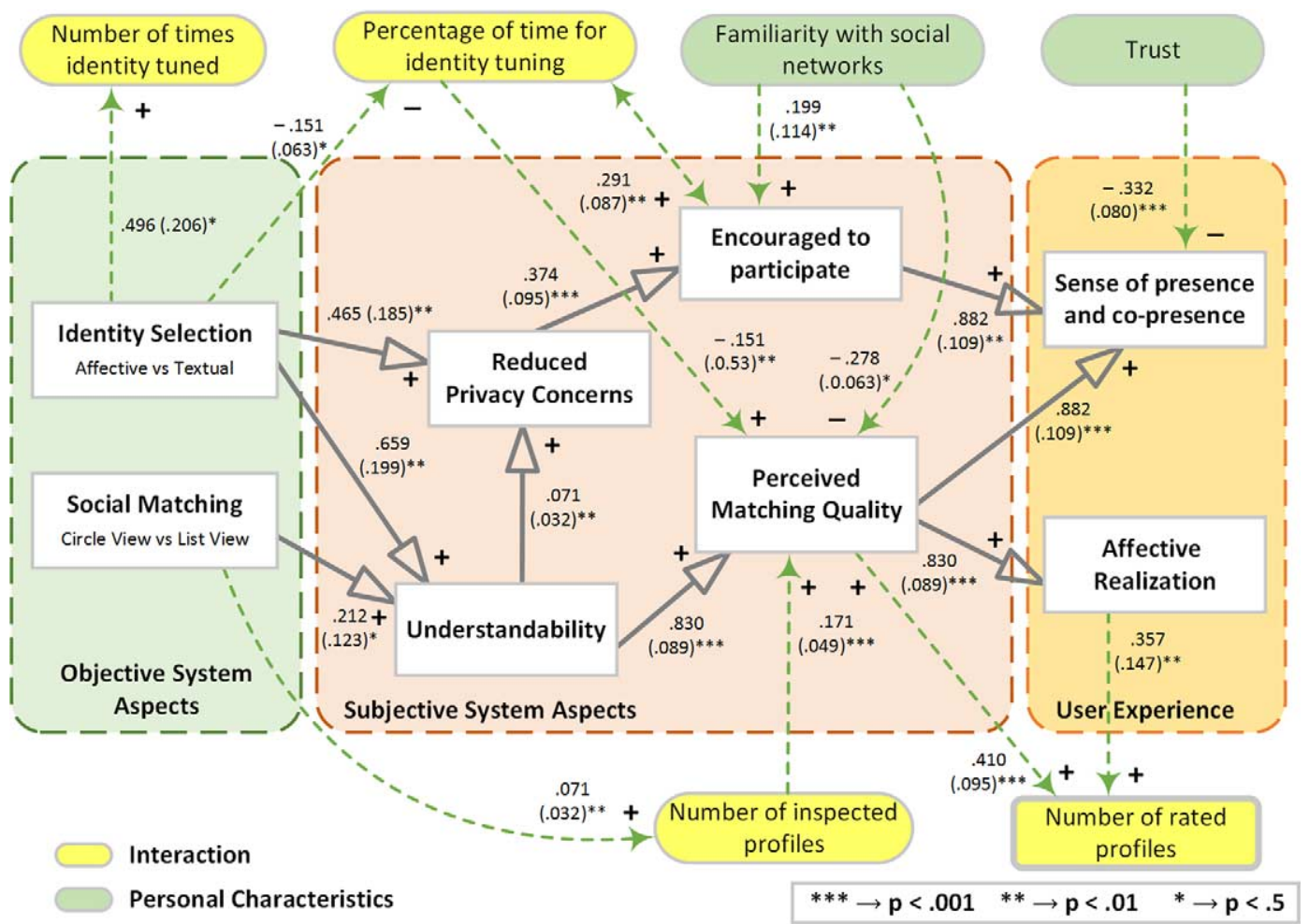

Fig. 4. The structural equation model. Numbers on the arrows represent the $\beta$ coefficients (and standard errors).

understandability of the system: the affective profile and social matching both have higher understandability (as compared to textual view). The understandability along with the identity selection have a positive effect on reducing the users' privacy concerns which leads to encouraging users to participate in the social network. The understandability is also in turn related to the perceived quality of matching. The perceived quality of matching and encouraging the user to participate result in making the sense of presence in the environment and the sense of co-presence with the others. In addition, the perceived quality of matching lead to realization of other humans affect. The marginal effects of the identity selection and social matching on these factors are presented in Fig. 5 (a, b, c, d). In this figure the effects of the "textual view" condition is set to zero.

The effects of this system on the user behavior which are mediated by identity selection and social matching mechanism also confirm the hypotheses. It directly influenced the number of inspected profiles by the user (Figs 5e and 5f). The participants took more time to inspect and review the profiles. They inspected more profiles in average (about 4.5 profile more). Which indicates a higher system effectiveness in the quality of matching process and recommendation. They also assigned rate to about 3 profiles more versus less than one in textual interface (they specified their desires to contact these profiles by rating). The intention to inspect the profiles has a positive influence on the perceived matching quality. Furthermore, the perceived quality of matching and affective realization both provide positive impact on the number of rated profiles which are direct effects of conveying affective cues.

In terms of behavior, the amount of time that a user tuned his identity increased with the effects of identity selection process. Conversely, this factor has a negative correlation with the percentage of time a user tuned its profile which may be due to the novelty of the controls used. The percentage of the time required for the identity tuning is also correlated with the encouraging the users to participate and the perceived quality of matching. It implies that the users are encouraged to adapt themselves with the other users.

The users who were familiar with the social networks were more eager to participate in the system; they also perceived lower matching quality due to the lower number of profiles exist in the system. 


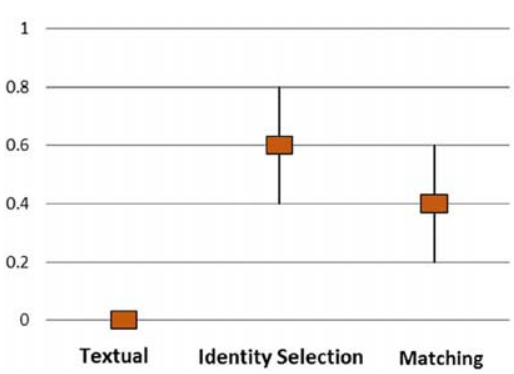

a) Understandability

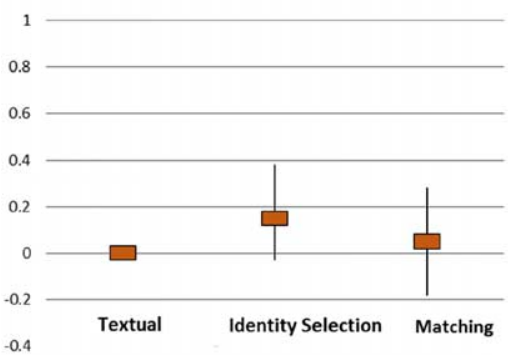

d) Affective realization

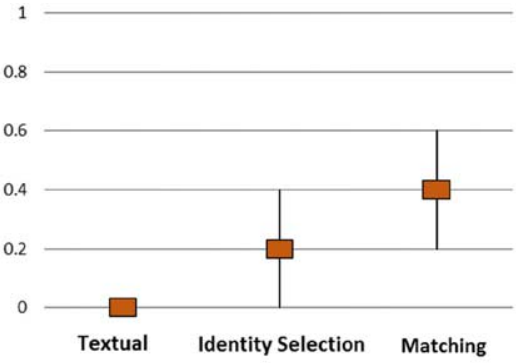

b) Perceived matching quality

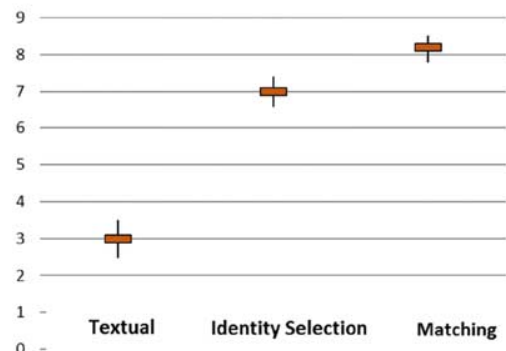

e) Number of inspected profiles

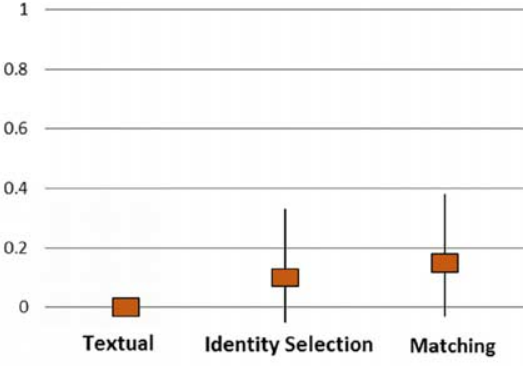

c) Sense of presence

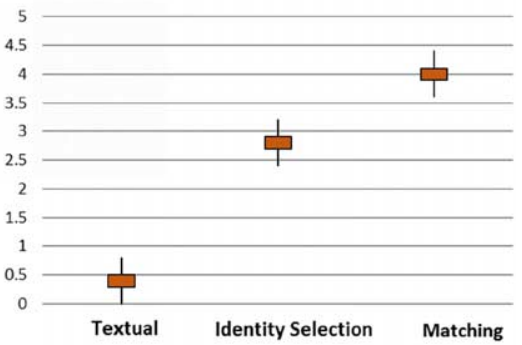

f) Number of rated profiles

Fig. 5. Marginal effects of the identity selection and social matching on the model factors.

\section{Discussion}

This paper described the communication mechanism of an ephemeral social network that is perfectly matched to the vehicular scenarios. A user interface is presented to facilitate social communication and convey the affective cues in this network. In this regard, different factors such as privacy and matching user mental model were considered. This architecture offers many advantages versus similar systems. Systems like [11-14] which benefit from profile introduction are not designed for vehicular environments and do not consider the interesting point of effective introduction. The proposed social network service overcomes this issue by using affective abstract identity.

In the following, the advantages of using such a system in communication between users based on the experiments are described in detail. It is also explained why the used mechanism are affective in achieving the systems goals.

\subsection{Affective identity selection}

This network is faced with different constraints like limited communication time and privacy concerns. Therefore, the identity selection method prepares the ground for rapid affective introduction. The evaluation shows that the abstract identity has a positive im- pact on the understandability of the users. Primarily because using visual characters and images for personality and preference selection are easier to understand. Moreover, the independence of these information to real identity and a proper understanding of this issue have a positive influence on reducing the user's privacy concerns. Increasing the understandability and reducing the privacy concerns both lead to more control over the system and encourage them to participate. This issue is clear by the user behavior in trying to select a suitable identity for the network and tune it. Finally, this issue along with relevant experience in finding appropriate contacts result in a sense of presence and copresence for the users. On the other hand, the novelty exists in this mechanism has a direct influence on the users to test different identities. Additionally, the identity selection functionality owes to proper understanding of the user, reduces the amount of time that identity is selected and tuned.

\subsection{Social matching and recommendation}

This investigation confirms that matching similar users based on the affective abstract identity leads to a sensible experience. This experience comes from both the quality perceived from the matching and satisfaction perceived from conveying the feelings and emotions; it is not necessarily a surprising result. Because 
in this way the amount of user effort to understand this information is reduced and his feeling is stimulated simultaneously. Therefore, comparing this system with the textual one is unbalanced in this respect. The model confirms that the derived results have been obtained actually from a better understanding of the matching mechanism. In fact, visual comparison and matching regarding the emotional state of both parties mediate itself on the user behavior. In the experiments, the matching mechanism used increased the number of inspected profiles (total effect: $\beta=0.279$, $\mathrm{p}=0.15$ ) and the number of inspected profiles increased the perceived matching quality (total effect: $\beta=0.376, \mathrm{p}=0.001)$.

More interestingly, this issue caused the tendency of the user to communicate with the others and inspired its affective connection to use this system. The witness of this claim is the user behavior in rating more profiles. (total effect: $\beta=0.565, \mathrm{p}=0.09$ ).

The scalability of the social matching scheme has been also evaluated by varying the number of profiles that may appear in the social network from 10 to 100 . Figure 6 reports the average of execution time to perform a social matching algorithm while varying the number of profiles. In other words, the graph shows the execution time of assigning a matching intensity which satisfies similarity of avatar and similarity of interests for a profile. The execution time linearly increases with the increase in the number of profiles. However, the increase is negligible with respect to the number of profiles. The execution time is so fast that the social matching scheme is transparent to the user.

\section{Conclusion}

In this paper, the opportunity of establishing an affective ephemeral social network in the heavy-traffic emerged in the metropolises is introduced. Such a network could easily benefit from the fun and entertainment. This network creates a space for communication between vehicles considering its unique characteristics and constraints. Accordingly, a service architecture is proposed through this paper to reflect the vehicular features and prepare the network for social applications. The architecture provides a social group management mechanism to facilitate information dissemination and management.

The advantage of an abstract identity for introduction and conveying emotions and preferences regarding the privacy is also highlighted in this architec-

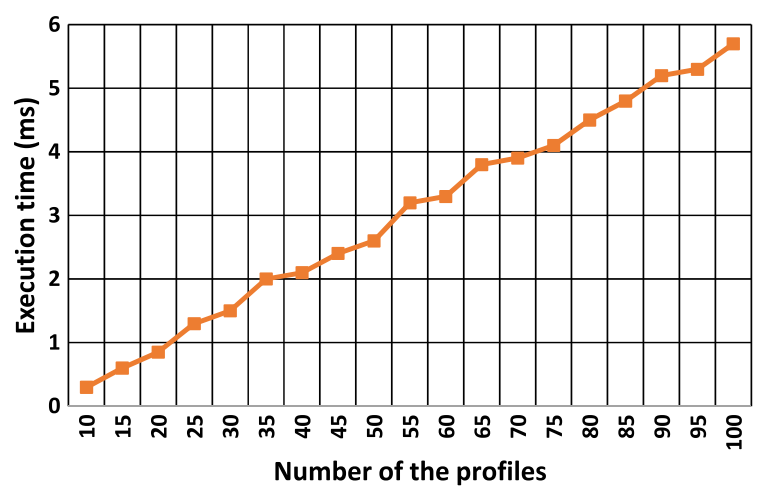

Fig. 6. Social matching execution times.

ture. This identity is expressive and match users' mental model. Furthermore, an affective social matching method is explored to recommend the desired contacts to the users.

Finally, an interface was designed to elicit the identities, personalities and preferences. The major aims were to encourage users to participate and convey affective cues. In order to evaluate these ideas, a prototype is developed and conducted a user study to investigate the solution and the impact of the objective system aspects over the subjective aspects, user experience and behavior of the user. The results show that there is a positive correlation between the affective identity and various aspects.

\section{References}

[1] I. Lequerica, M. García Longaron and P.M. Ruiz, Drive and share: efficient provisioning of social networks in vehicular scenarios, IEEE Communications Magazine 48(11) (2010), 90-97.

[2] S.W. Littlejohn and K.A. Foss, Theories of Human Communication, Cengage Learning, 2008.

[3] E. Schoch, F. Kargl, M. Weber and T. Leinmuller, Communication patterns in VANETs, IEEE Communications Magazine 46(11) (2008), 119-125.

[4] J. Rao and T.J. Giuli, Evaluating vehicular ad hoc networks for group applications, in: 2011 IEEE International Conference on Pervasive Computing and Communications Workshops (PERCOM Workshops), Seattle, WA, United States, 2011, pp. 594599.

[5] A. Rahim, Z.S. Khan and F.B. Muhaya, Performance evaluation of video streaming in vehicular adhoc network, in: Information Security and Assurance, Springer, Berlin, Heidelberg, 2010, pp. 220-224.

[6] O.K. Tonguz and M. Boban, Multiplayer games over vehicular ad hoc networks: a new application, Ad Hoc Networks 8(5) (2010), 531-543. 
[7] J. Jiang, H.-z. Chen and Y. Fang, Distributed autonomous navigation system based on VANET [J], Computer Engineering 2 (2009), 98

[8] R. Schroeter, A. Rakotonirainy and M. Foth, The social car: new interactive vehicular applications derived from social media and urban informatics, in: Proc. of the 4th International Conference on Automotive User Interfaces and Interactive Vehicular Applications, Portsmouth, NH, United States, 2012, pp. 107-110.

[9] F. Alt, D. Kern, F. Schulte, B. Pfleging, A.S. Shirazi and A. Schmidt, Enabling micro-entertainment in vehicles based on context information, in: Proc. of the 2nd International Conference on Automotive User Interfaces and Interactive Vehicular Applications, Pittsburgh, PA, United States, 2010, pp. 117124.

[10] U. Lee, J. Lee, J.-S. Park and M. Gerla, FleaNet: a virtual market place on vehicular networks, IEEE Transactions on Vehicular Technology 59(1) (2010), 344-355.

[11] D. Bottazzi, R. Montanari and A. Toninelli, Context-aware middleware for anytime, anywhere social networks, IEEE Intelligent Systems 22(5) (2007), 23-32.

[12] A. Gupta, A. Kalra, D. Boston and C. Borcea, MobiSoC: a middleware for mobile social computing applications, Mobile Networks and Applications 14(1) (2009), 35-52.

[13] A.-K. Pietiläinen, E. Oliver, J. LeBrun, G. Varghese and C. Diot, MobiClique: middleware for mobile social networking, in: Proc. of the 2nd ACM Workshop on Online Social Networks, New York, NY, USA, 2009, pp. 49-54.

[14] A. Toninelli, A. Pathak and V. Issarny, Yarta: a middleware for managing mobile social ecosystems, in: Advances in Grid and Pervasive Computing, Springer, 2011, pp. 209-220.

[15] P. Wallace and J. Maryott, The impact of avatar selfrepresentation on collaboration in virtual worlds, Innovate: Journal of Online Education 5(5) (2009), p. 2009.

[16] J.G. Hamilton, Identifying with an avatar: a multidisciplinary perspective, in: Proc. of the Cumulus Conference: $38^{\circ}$ South: Hemispheric Shifts Across Learning, Teaching and Research, Swinburne University of Technology and and RMIT University, 2009.

[17] Y. Sung, J.H. Moon and J.-S. Lin, Actual self vs. avatar self: the effect of online social situation on self-expression, Journal For Virtual Worlds Research 4(1) (2011).

[18] P.R. Messinger, X. Ge, E. Stroulia, K. Lyons, K. Smirnov and $\mathrm{M}$. Bone, On the relationship between my avatar and myself, Journal For Virtual Worlds Research 1(2) (2008).

[19] M. Prince, Virtual identities from virtual environments, in: Digital Media and Advertising: User Generated Content Consumption, M.S. Eastin, T. Daugherty and N.M. Burns, eds, Information Science Reference, New York, 2011, pp. 335-44.

[20] J.F. Chen, C.A. Warden, D. Wen-Shung Tai, F.-S. Chen and C.-Y. Chao, Level of abstraction and feelings of presence in virtual space: business english negotiation in open wonderland, Computers \& Education 57(3) (2011), 2126-2134.

[21] G. Bente, S. Rüggenberg, N.C. Krämer and F. Eschenburg, Avatar-mediated networking: increasing social presence and interpersonal trust in net-based collaborations, Human Communication Research 34(2) (2008), 287-318.

[22] D. Rousseau and B. Hayes-Roth, A social-psychological model for synthetic actors, in: Proc. of the Second International Conference on Autonomous Agents, New York, NY, USA, 1998, pp. 165-172.
[23] A. Ortony, The Cognitive Structure of Emotions, Cambridge University Press, 1990.

[24] C.L. Lisetti, Personality, affect and emotion taxonomy for socially intelligent agents, in: FLAIRS Conference, 2002, pp. 397-401.

[25] R.W. Picard, What Does It Mean for a Computer to "Have" Emotions, MIT Press, Cambridge, 2003.

[26] S.D. Gosling, P.J. Rentfrow and W.B. Swann Jr., A very brief measure of the Big-Five personality domains, Journal of Research in Personality 37(6) (2003), 504-528.

[27] J.A. Russell, M. Lewicka and T. Niit, A cross-cultural study of a circumplex model of affect, Journal of Personality and Social Psychology 57(5) (1989), 848.

[28] J.A. Russell and A. Mehrabian, Evidence for a three-factor theory of emotions, Journal of Research in Personality 11(3) (1977), 273-294.

[29] G. Valenza and E.P. Scilingo, Emotions and mood states: modeling, elicitation, and classification, in: Autonomic Nervous System Dynamics for Mood and Emotional-State Recognition, Springer, 2014, pp. 9-21.

[30] J. Broekens and W.-P. Brinkman, AffectButton: a method for reliable and valid affective self-report, International Journal of Human-Computer Studies 71(6) (2013), 641667.

[31] M.S. Kakkasageri and S.S. Manvi, Information management in vehicular ad hoc networks: a review, Journal of Network and Computer Applications 39 (2014), 334-350.

[32] A. Reyes, C. Barrado, M. López and C. Excelente, Vehicle density in VANET applications, Journal of Ambient Intelligence and Smart Environments 6(4) (2014), 469-481.

[33] M. Koubek, S. Rea and D. Pesch, Event suppression for safety message dissemination in VANETs, in: 2010 IEEE 71st Vehicular Technology Conference (VTC 2010-Spring), Taipei, Taiwan, 2010, pp. 1-5.

[34] P. Fan, J. Haran, J. Dillenburg and P.C. Nelson, Traffic model for clustering algorithms in vehicular ad-hoc networks, in: Proc. of CCNC, Las Vegas, Nevada, United State, 2006, pp. $168-172$.

[35] C. Lochert, B. Scheuermann and M. Mauve, Probabilistic aggregation for data dissemination in VANETs, in: Proc. of the Fourth ACM International Workshop on Vehicular ad Hoc Networks, Montreal, QC, Canada, 2007, pp. 1-8.

[36] K. Dar, M. Bakhouya, J. Gaber and M. Wack, Evaluating information dissemination approaches in VANETs, in: Proc. 7 th ACM International Conference on Pervasive Services (ICPS 2010), July, Berlin, Germany, 2010, pp. 120-125.

[37] N. Kumar, R. Iqbal and N. Chilamkurti, Capacity and loadaware service discovery with service selection in peer-to-peer grids, Future Generation Computer Systems 28(7) (2012), 1090-1099.

[38] N. Kumar, R. Iqbal, S. Misra and J.J.P.C. Rodrigues, Bayesian coalition game for contention-aware reliable data forwarding in vehicular mobile cloud, Future Generation Computer Systems, DOI:10.1016/j.future.2014.10.013.

[39] P. Fan, Improving broadcasting performance by clustering with stability for inter-vehicle communication, in: IEEE 65th Vehicular Technology Conference, 2007, VTC2007-Spring, Dublin, Ireland, 2007, pp. 2491-2495.

[40] T.K. Forde, L.E. Doyle and D. O'Mahony, Ad hoc innovation: distributed decision making in ad hoc networks, IEEE Communications Magazine 44(4) (2006), 131-137. 
[41] M.M. Wasko and S. Faraj, Why should I share? Examining social capital and knowledge contribution in electronic networks of practice, MIS Quarterly 29(1) (2005), 35-57.

[42] A. Garyfalos and K.C. Almeroth, Coupons: a multilevel incentive scheme for information dissemination in mobile networks, IEEE Transactions on Mobile Computing 7(6) (2008), 792804.

[43] S. Gangwar, Mobile ad hoc network: a comprehensive study and survey on intrusion detection, International Journal of Engineering Research and Applications (IJERA) 2(1) (2012), $607-612$.

[44] S. Brewster and M. Dunlop, Proc. of Mobile Human-Computer Interaction-Mobile HCI 2004: 6th International Symposium, Glasgow, UK, September 13-16, 2004, Vol. 3160, Springer, 2004.

[45] Y. Rogers, H. Sharp and J. Preece, Interaction Design: Beyond Human-Computer Interaction, John Wiley \& Sons, Inc, 2011.

[46] P. Bellavista, R. Montanari and S.K. Das, Mobile social networking middleware: a survey, Pervasive and Mobile Computing 9(4) (2013), 437-453.

[47] D. Judith, Being Real: Questions of Tele-Identity, MIT Press, Cambridge, MA, USA, 2001.

[48] J.S. Donath, Identity and deception in the virtual community, Vol. 1996, 1999.

[49] S. Turkle, in: Life on the Screen: Identity in the Age of the Internet, Literature and History, Vol. 6, 1997, pp. 117-118.

[50] E. Weber and E. Johnson, Constructing preferences from memory, in: The Construction of Preference, S. Lichtenstein and P. Slovic, eds, Cambridge University Press, New York, NY, 2006, pp. 397-410.

[51] E.J. Johnson, M. Steffel and D.G. Goldstein, Making better decisions: from measuring to constructing preferences, Health Psychology 24(4S) (2005), S17.

[52] G. Carenini and D. Poole, Constructed preferences and valuefocused thinking: implications for AI research on preference elicitation, in: AAAI-02 Workshop on Preferences in AI and CP: Symbolic Approaches, Alberta, Canada, 2002, pp. 1-10.

[53] L. Terveen and D.W. McDonald, Social matching: a framework and research agenda, ACM Transactions on Computer-Human Interaction (TOCHI) 12(3) (2005), 401-434.

[54] J. Paay and J. Kjeldskov, Understanding the user experience of location-based services: five principles of perceptual organisation applied, Journal of Location Based Services 2(4) (2008), 267-286.

[55] G.W. Fischer, Z. Carmon, D. Ariely and G. Zauberman, Goalbased construction of preferences: task goals and the prominence effect, Management Science 45(8) (1999), 1057-1075.

[56] E.J. Johnson, M. Steffel and D.G. Goldstein, Making better decisions: from measuring to constructing preferences, Health Psychology 24(4, Suppl) (2005), S17-S22.
[57] T. Kramer, The effect of measurement task transparency on preference construction and evaluations of personalized recommendations, Journal of Marketing Research 44(2) (2007), 224-233.

[58] C. Sedikides, D. Ariely and N. Olsen, Contextual and procedural determinants of partner selection: of asymmetric dominance and prominence, Social Cognition 17(2) (1999), 118-139.

[59] M.L. Sichitiu and M. Kihl, Inter-vehicle communication systems: a survey, IEEE Communications Surveys \& Tutorials 10(2) (2008), 88-105.

[60] W. Chen, R.K. Guha, T.J. Kwon, J. Lee and Y.Y. Hsu, A survey and challenges in routing and data dissemination in vehicular ad hoc networks, Wireless Communications and Mobile Computing 11(7) (2011), 787-795.

[61] F. Li and Y. Wang, Routing in vehicular ad hoc networks: a survey, IEEE Vehicular Technology Magazine 2(2) (2007), 1222.

[62] T.L. Willke, P. Tientrakool and N.F. Maxemchuk, A survey of inter-vehicle communication protocols and their applications, IEEE Communications Surveys \& Tutorials 11(2) (2009), 3 20.

[63] P. Fan, P. Sistla and P. Nelson, Theoretical analysis of a directional stability-based clustering algorithm for VANETs, in: Proc. of the Fifth ACM International Workshop on VehiculAr Inter-NETworking, 2008, pp. 80-81.

[64] C.R. Lin and M. Gerla, Adaptive clustering for mobile wireless networks, IEEE Journal on Selected Areas in Communications 15(7) (1997), 1265-1275.

[65] D. Jiang and L. Delgrossi, IEEE 802.11 p: towards an international standard for wireless access in vehicular environments, in: IEEE Vehicular Technology Conference, 2008, VTC Spring 2008, Singapore, 2008, pp. 2036-2040.

[66] A. Pommeranz, J. Broekens, P. Wiggers, W.-P. Brinkman and C.M. Jonker, Designing interfaces for explicit preference elicitation: a user-centered investigation of preference representation and elicitation process, User Modeling and User-Adapted Interaction 22(4-5) (2012), 357-397.

[67] P. Pu, L. Chen and R. Hu, Evaluating recommender systems from the user's perspective: survey of the state of the art, User Modeling and User-Adapted Interaction 22(4-5) (2012), 317 355.

[68] D. Preuveneers and P. Novais, A survey of software engineering best practices for the development of smart applications in Ambient Intelligence, Journal of Ambient Intelligence and Smart Environments 4(3) (2012), 149-162.

[69] B.P. Knijnenburg, M.C. Willemsen, Z. Gantner, H. Soncu and C. Newell, Explaining the user experience of recommender systems, User Modeling and User-Adapted Interaction 22(45) (2012), 441-504. 